\title{
MYC and hsa-miRNA-423-5p as biomarkers in nasopharyngeal carcinoma revealed by miRNA-mRNA-pathway network integrated analysis
}

\author{
HUAN TIAN* ${ }^{*}$, SHICAI CHEN*, CAIYUN ZHANG, MENG LI and HONGLIANG ZHENG \\ Department of Otolaryngology-Head and Neck Surgery, Changhai Hospital, \\ The Second Military Medical University, Shanghai 200433, P.R. China
}

Received March 7, 2016; Accepted February 21, 2017

DOI: $10.3892 / \mathrm{mmr} .2017 .6696$

\begin{abstract}
The present study was performed to identify the dysregulated microRNAs (miRNAs/miRs) and mRNAs, and enriched pathways involved in nasopharyngeal carcinoma (NPC) through the establishment of an miRNA-mRNA-pathways network. mRNA and miRNA expression profiles were collected from the European Molecular Biology Laboratory-European Bioinformatics Institute. Differentially expressed genes and differentially expressed miRNA were selectively screened using the metaDE package. Following prediction of the risk genes and pathway pairs involved in NPC, an miRNA-mRNA-pathway network was constructed by merging the miRNA-mRNA pairs, the mRNA-pathway pairs and the mRNA-mRNA pairs. The miRNA and mRNA biomarkers, as well as the functional pathway pairs, were identified in the network analysis, based on the topological properties of nodes in the network. Additionally, 10 -fold cross-validation was performed to evaluate the performance of the selected risk genes and their corresponding miRNA in NPC by calculating the area under the curve (AUC). In total, 99 upregulated and 841 downregulated genes, and 192 upregulated and 26 downregulated miRNAs were identified. The miRNA-mRNA-pathway network was established using 403 miRNA-mRNA pairs, including 40 miRNAs and 302 risk genes, as well as 22 prominent pathway pairs. Network analysis demonstrated that v-myc avian myelocytomatosis viral oncogene homolog (MYC) and hsa-miR-423-5p were the mRNA and miRNA signatures for NPC, respectively. The AUC of these biomarkers for NPC was 0.7568 and 0.7798 , respectively. Additionally, the focal adhesion pair pathway in
\end{abstract}

Correspondence to: Dr Hongliang Zheng, Department of Otolaryngology-Head and Neck Surgery, Changhai Hospital, The Second Military Medical University, 168 Changhai Road, Yangpu, Shanghai 200433, P.R. China

E-mail: zhengdhdrrf@sina.com

${ }^{*}$ Contributed equally

Key words: nasopharyngeal carcinoma, differentially expressed genes, microRNA, microRNA-mRNA-pathway pair network cancer was identified to be associated with NPC. MYC and hsa-miR-423-5p have been identified to be critical biomarkers in NPC as revealed by miRNA-mRNA-pathway network integrated analysis, suggesting a direction for further research into the diagnosis and treatment of NPC.

\section{Introduction}

Nasopharyngeal carcinoma (NPC) is a common head and neck cancer that arises from the nasopharynx epithelium. Although excellent local control can be achieved with advances in multimodal therapy, the poor prognosis of NPC with frequent tumor recurrence and distant metastases obstructs long-term patient survival (1). As previously described (2), genetic susceptibility, as well as environmental factors and Epstein-Bar virus (EBV) infection are the three primary etiologic factors of NPC. However, the molecular mechanism of its pathogenesis is still unclear (2). Exploring the underlying molecular mechanisms of NPC involved in its pathogenesis and progression may improve NPC diagnosis and therapy.

MicroRNAs (miRNAs/miRs), a class of small non-coding RNAs, have emerged as post-transcriptional regulators of gene expression by binding to the untranslated region during various biological processes (3). There are an increasing number of studies concerning miRNA-dysregulation in cancers including NPC (4-6). As noted previously, miRNAs including hsa-miR-141, hsa-miR-138, hsa-miR-200a and hsa-miR-26a are altered in NPC (7-9). However, these studies were focused on experimental validation for selected outlier miRNAs. Systematic computational methods, which integrate miRNA regulatory data and gene expression profiling data, provide an effective tool to understand the role of miRNA with respect to modules or interactions (10). A conceptual miRNA regulation module has been proposed based on the multiple-to-multiple relationship between miRNAs and their target genes (11). Due to the co-regulatory effects of miRNAs on the same genes, abnormal miRNAs may exhibit increased functional synergism (12). This concept has been investigated in cancer research and several candidate abnormal miRNAs or miRNA regulatory modules have been identified (13-15).

A change in gene expression levels is observed in tumor samples, which is believed to be an important mechanism 
of tumorigenesis and pathogenicity. Gene expression is not isolated and there is an association between gene expression and gene function, leading to a tendency to study modular expression associated with function $(16,17)$. Gene regulatory network modules are able to be organized into lower dimensional function modules, which have marked biological significance (18). The functional pairs with highly correlating expression trends may be obtained through analyzing the expression correlation of these function modules. Furthermore, the functional pairs associated with NPC may be screened using hypergeometric testing.

In the present study, the miRNA and mRNA expression profiles were integrated, as well as functional pairs, to construct an miRNA-mRNA-pathway network to evaluate the candidate risk factors associated with NPC and the miRNA biomarkers.

\section{Materials and methods}

Data collection and processing. mRNA and miRNA expression profiles were collected and extracted from the European Molecular Biology Laboratory-European Bioinformatics Institute (EMBL-EBI; www.ebi.ac.uk). 'NPC' was selected as the key word to search gene expression profiles, within which the study type was limited to array expression profiling and the species was limited to human. The following datasets were excluded: i) Tissue samples were cell samples; ii) samples were not NPC tissue; iii) the datasets did not include normal controls; and iv) the microarray platform did not include sufficient intersecting data with other platforms.

The normalized data from included microarrays were downloaded and the raw probe-level data in the mRNA expression files were converted into expression measures. For each sample, the expression values for a given gene were reduced to a single value by taking the average expression value of all probes.

Differentially expressed gene (DEG) and differentially expressed miRNA (DEmiR) analysis. The metaDE package (19) in $\mathrm{R}$ language was used to screen out the DEGs and DEmiRs between NPC samples and normal controls. $\mathrm{P}<0.05$ and $\log _{2}$ fold change $(\mathrm{FC}) \mid>1.0$ were set as thresholds.

Prediction risk genes for NPC. The target genes of DEmiRs were obtained from three different databases: miRecords (version 3; http://c1.accurascience.com/miRecords/), miRTarbase (release 2.5; http://mirtarbase.mbc.nctu.edu.tw/) and Tarbase (version 5.0; http://diana.imis.athena-innovation.gr/ DianaTools/index.php? $\mathrm{r}=$ tarbase/index/). The target genes predicted by the above three databases were merged to one uniform non-redundant set. The intersection of DEGs and the target genes of DEmiRs were considered to be the risk genes of NPC for further analysis.

Pathway enrichment analysis. Gene Ontology (GO) (20) and Kyoto Encyclopedia of Genes and Genomes (KEGG) (21) were performed for biological processes analysis and functional pathways enrichment of risk genes by Database for Annotation, Visualization and Integrated Discovery (DAVID) (22), which is an integrated biological knowledge base and effective analytic tool to systematically extract biological information and explore the biological meanings behind abundant lists of genes or proteins. $\mathrm{P}<0.05$ was set as the threshold value.

Pathway expression profile construction. All known signaling pathways and pathway genes in humans were downloaded from the PATHWAY database in KEGG. The expression levels for each pathway in the selected samples were evaluated by Median, using the following formula: $\operatorname{Path}_{i k}=\operatorname{Median}_{k}\left(g_{l}\right.$, $\left.g_{2}, g_{3}, \ldots, g_{n}\right)$, where Path $_{i k}$ indicates the expression level of pathway $i$ in sample $k$; and $g_{1}, g_{2}, g_{3}, \ldots, g_{n}$ indicate the expression level of all genes from the pathway in sample $k$ (23). Therefore, the pathway expression profile was established based on the expression level of pathways in selected samples by calculating the Median.

Expression correlation analysis among signaling pathways. The pathogenesis of NPC is associated with abnormal expression of multiple signaling pathways. The expression correlation between pathway pairs was calculated to identify the important pathway. The pathways were considered critical pathways if they were highly correlated in both NPC and normal samples.

The expression correlation between two pathways in all samples (NPC samples and control samples) were evaluated by calculating the Pearson's correlation coefficient:

$$
P_{X, Y}=\frac{\sum(X-\bar{X})(Y-\bar{Y})}{\sqrt{\sum(X-\bar{X})^{2} \sum(Y-\bar{Y})^{2}}}
$$

Where $P_{X, Y}$ indicates the expression coefficient between pathway $X$ and pathway $Y$ among all samples; and $\bar{X}$ and $\bar{Y}$ and indicate the mean expression level of pathway $X$ and pathway $Y$ among all samples, respectively. The pathway pairs with coefficient $>0.5$ were considered to be candidates.

The pathway pairs involved in NPC were screened using the hypergeometric test as follows:

$$
p=1-\sum_{i=0}^{k-1} \frac{C_{M}^{i} C_{N-M}^{n-i}}{C_{N}^{n}}
$$

Where $n$ indicates the same risk genes between two couple pathways; $N$ indicates all genes between two couple pathways; and $M$ indicates all risk genes between two couple pathways. Any pathway pair with $\mathrm{P}<0.05$ was considered to be a prominent pathway pair in NPC.

Establishment of miRNA-mRNA-pathway network. The same genes in prominent pathway pairs were selected and the corresponding mRNA-pathway pairs were obtained. Furthermore, mRNA-mRNA pairs were extracted from the Human Protein Reference Database (HPRD; www.hprd.org). Afterwards, the miRNA-mRNA pairs, the mRNA-pathway pairs and the mRNA-mRNA pairs were constructed into a miRNA-mRNA-pathway network.

The nodes in the network included miRNA, risk genes and pathway pairs, while the edges represented the miRNA-mRNA relationship pairs as well as mRNA-pathway pairs. This 
Table I. mRNA and miRNA datasets information for NPC.

\begin{tabular}{|c|c|c|c|c|}
\hline Microarray & Microarray type & Platform & No. of NPC samples & No. of normal control samples \\
\hline GSE12452 & mRNA & GPL570 & 31 & 10 \\
\hline GSE13597 & mRNA & GPL96 & 25 & 3 \\
\hline GSE34573 & mRNA & GPL570 & 15 & 3 \\
\hline GSE53819 & mRNA & GPL6480 & 18 & 18 \\
\hline GSE32960 & miRNA & GPL14722 & 312 & 18 \\
\hline GSE46172 & miRNA & GPL16770 & 4 & 4 \\
\hline
\end{tabular}

miRNA, microRNA; NPC, nasopharyngeal carcinoma.

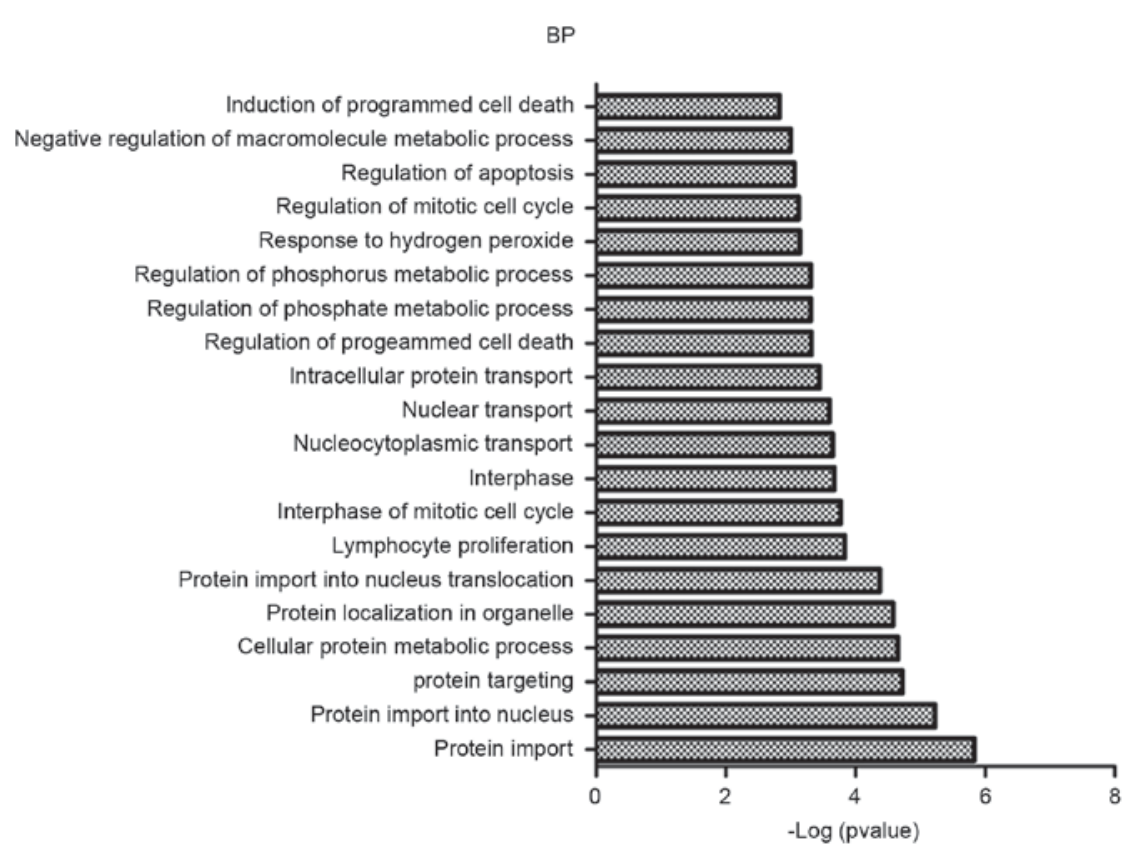

Figure 1. Gene Ontology annotation of biological processes for risk genes. Vertical axis indicates the enriched BP; horizontal axis indicates the level of enrichment. BP, biological processes.

miRNA-mRNA-pathway network reflects not only the regulatory association between miRNA and risk genes, but also indicates the regulatory function of miRNA.

The risk genes involved in NPC were further screened by analyzing the topological properties of the miRNA-mRNA-pathway network using the network analysis plugin in Cytoscape (24) in combination with the consideration of the regulatory function of miRNA. These topological properties included degree, average shortest path length, closeness centrality, betweenness centrality and topological coefficient. The 10-fold cross-validation was used to measure the performance of the selected risk genes and their corresponding miRNA for the NPC by calculating the AUC (area under the curve) of the receiver-operating characteristic (ROC) curve.

\section{Results}

Raw data. A total of 43 relevant gene expression profiles were identified in EMBL-EBI, of which 36 were excluded. A total of six gene expression profiles (four for mRNA expression profiles and two for miRNA expression profiles) were included in the present study (Table I).

Identification of DEGs and DEmiRs in NPC. A total of 1540 DEGs were selected between NPC samples and normal controls. Among these DEGs, 699 genes were upregulated and 841 genes were downregulated. A total of 218 DEmiRs were collected, including 192 upregulated and 26 downregulated miRNAs.

Prediction risk genes for NPC. A total of 3,641 miRNA-target gene pairs were identified for NPC-specific DEmiRs. The intersection of DEGs and the target genes of DEmiRs were considered to be the risk genes of NPC for further analysis. A total of 403 miRNA-mRNA pairs including 40 miRNA and 302 risk genes were obtained.

Significant function and pathways of risk genes. GO and KEGG pathway enrichment analyses were performed for 
Table II. Topological properties of 22 prominent pathways pairs in the miRNA-risk gene-pathway network.

\begin{tabular}{|c|c|c|c|c|c|}
\hline Pathway pair & $\begin{array}{l}\text { Topological } \\
\text { Coefficient }\end{array}$ & $\begin{array}{l}\text { Average shortest } \\
\text { path length }\end{array}$ & $\begin{array}{l}\text { Betweenness } \\
\text { centrality }\end{array}$ & $\begin{array}{l}\text { Closeness } \\
\text { centrality }\end{array}$ & Degree \\
\hline Pathways in cancer_focal adhesion ${ }^{a}$ & 0.1742 & 2.8952 & 0.0268 & 0.3454 & 24 \\
\hline Proteoglycans in cancer_pathways in cancer & 0.1854 & 2.8754 & 0.0243 & 0.3478 & 21 \\
\hline Wnt signaling pathway_pathways in cancer & 0.2162 & 2.9207 & 0.0109 & 0.3424 & 19 \\
\hline Epstein-barr virus infection_htlv-i infection & 0.2144 & 3.1473 & 0.0107 & 0.3177 & 17 \\
\hline Pathways in cancer_colorectal cancer & 0.2280 & 2.9292 & 0.0068 & 0.3414 & 17 \\
\hline Htlv-i infection_colorectal cancer & 0.2419 & 2.9688 & 0.0059 & 0.3368 & 15 \\
\hline $\begin{array}{l}\text { Viral carcinogenesis _herpes simplex } \\
\text { infection }\end{array}$ & 0.1946 & 2.9972 & 0.0128 & 0.3336 & 15 \\
\hline Wnt signaling pathway_colorectal cancer & 0.2637 & 2.9745 & 0.0054 & 0.3362 & 13 \\
\hline $\begin{array}{l}\text { Wnt signaling pathway _choline } \\
\text { metabolism in cancer }\end{array}$ & 0.2876 & 3.1671 & 0.0025 & 0.3157 & 11 \\
\hline Cell cycle_small cell lung cancer & 0.2909 & 3.0623 & 0.0026 & 0.3265 & 9 \\
\hline Colorectal cancer_hepatitis b & 0.3575 & 3.1983 & 0.0013 & 0.3127 & 8 \\
\hline Rap1 signaling pathway_adherens junction & 0.2609 & 3.2720 & 0.0026 & 0.3056 & 8 \\
\hline Bladder cancer_calcium signaling pathway & 0.2912 & 3.0850 & 0.0017 & 0.3242 & 7 \\
\hline Bladder cancer_cell cycle & 0.3544 & 3.1841 & 0.0008 & 0.3141 & 7 \\
\hline Bladder cancer_small cell lung cancer & 0.3383 & 3.2465 & 0.0004 & 0.3080 & 7 \\
\hline $\begin{array}{l}\text { Chemokine signaling pathway _viral } \\
\text { myocarditis }\end{array}$ & 0.2612 & 3.2238 & 0.0046 & 0.3102 & 7 \\
\hline Ecm-receptor interaction_amoebiasis & 0.3029 & 3.3541 & 0.0014 & 0.2981 & 7 \\
\hline P53 signaling pathway _cell cycle & 0.3794 & 3.2125 & 0.0007 & 0.3113 & 6 \\
\hline Shigellosis_pancreatic cancer & 0.3690 & 3.2380 & 0.0010 & 0.3088 & 6 \\
\hline NF-kappa b signaling pathway_tuberculosis & 0.3273 & 3.6232 & 0.0013 & 0.2760 & 5 \\
\hline Salmonella infection_shigellosis & 0.4872 & 3.3399 & 0.0004 & 0.2994 & 4 \\
\hline Bile secretion_salivary secretion & 0.5000 & 3.7960 & 0.0002 & 0.2634 & 2 \\
\hline
\end{tabular}

${ }^{a}$ Highest degree. HTLV-I, human T-lymphotropic virus 1; Rap1, DNA-binding protein RAP1; ECM, extracellular matrix.

risk genes, and the results are presented in Figs. 1 and 2. According to the results, risk genes were enriched in multiple GO categories, primarily including protein import, protein targeting and protein localization in an organelle, etc. Simultaneously, the risk genes were enriched in several signaling pathways, including colorectal cancer pathways, Wnt signaling pathways and mitogen-activated protein kinase signaling pathways.

Prediction pathways for NPC. A total of 293 human signaling pathways were downloaded from the PATHWAY database in KEGG. The pathway expression profile was obtained by calculating the $P a t h_{i k}$ of each pathway in each sample. Following calculation of the Pearson's correlation coefficient, 473 highly-correlated pathway pairs were acquired. A total of 22 prominent pathway pairs were subsequently selected using the hypergeometric test, all of which were associated with risk genes (Table II).

Construction of miRNA-mRNA-pathway network. There were 69 mRNA-mRNA pairs extracted from HPRD with 38 risk genes. Following the merging of the miRNA-mRNA relationship pairs, the mRNA-pathway pairs and the mRNA-mRNA

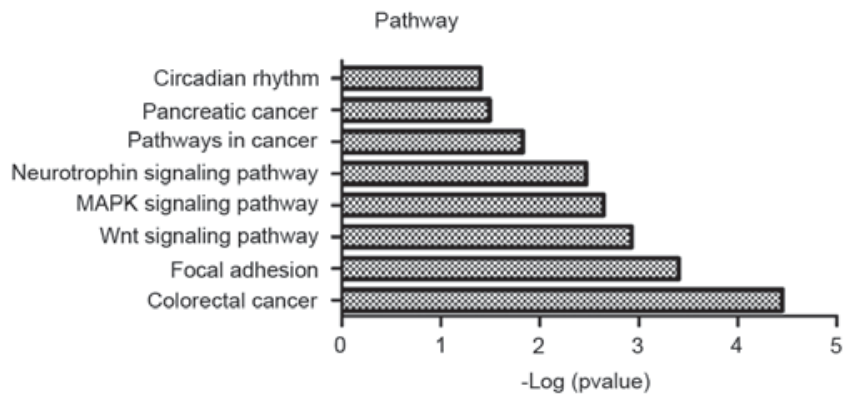

Figure 2. Kyoto Encyclopedia of Genes and Genomes enrichment analyses for risk genes. Vertical axis indicates the enriched pathways; horizontal axis indicates the level of enrichment. MAPK, mitogen-activated protein kinase.

pairs, the miRNA-mRNA-pathway network was constructed. A total of 702 relationship pairs and 360 nodes were discovered (Fig. 3).

Functional analysis of miRNA-mRNA-pathway network. Following analysis of the topological properties of 22 prominent pathways pairs in the miRNA-mRNA-pathway network, three pathway pairs were associated with risk genes, including 
Table III. Topological properties of 15 selected risk genes with highest degrees in the miRNA-risk gene-pathway network.

\begin{tabular}{llccc}
\hline Risk gene & $\begin{array}{c}\text { Topological } \\
\text { coefficient }\end{array}$ & $\begin{array}{c}\text { Average shortest } \\
\text { path length }\end{array}$ & $\begin{array}{c}\text { Betweenness } \\
\text { centrality }\end{array}$ & $\begin{array}{c}\text { Closeness } \\
\text { centrality }\end{array}$ \\
\hline MYC & 0.0026 & 2.0741 & 0.3792 & 0.4821 \\
TP53 & 0.0031 & 2.1036 & 0.2838 & 0.4754 \\
JUN & 0.0063 & 2.2764 & 0.0923 & 0.4393 \\
EGFR & 0.0065 & 2.528 & 0.1124 & 0.3956 \\
BIRC5 & 0.0074 & 2.3675 & 0.0784 & 0.4224 \\
TBP & 0.0075 & 2.429 & 0.065 & 0.4117 \\
MAPK8 & 0.009 & 2.3637 & 0.0543 & 0.4231 \\
SKP2 & 0.01 & 2.4516 & 0.044 & 0.4079 \\
JAK2 & 0.0103 & 2.668 & 0.0513 & 0.3748 \\
FGF2 & 2.5393 & 0.0467 & 0.3938 \\
RAC1 & 0.0106 & 0.0708 & 0.357 \\
BCL2 & 0.0106 & 2.8012 & 0.0418 & 195 \\
CDC42 & 0.0108 & 2.4651 & 0.0419 & 170 \\
EZR & 0.0126 & 0.7745 & 0.023 & 156 \\
PSMD12 & 0.0126 & 2.7924 & 2.9105 & 0.4057 \\
\hline
\end{tabular}

${ }^{\mathrm{a}}$ Highest degree.

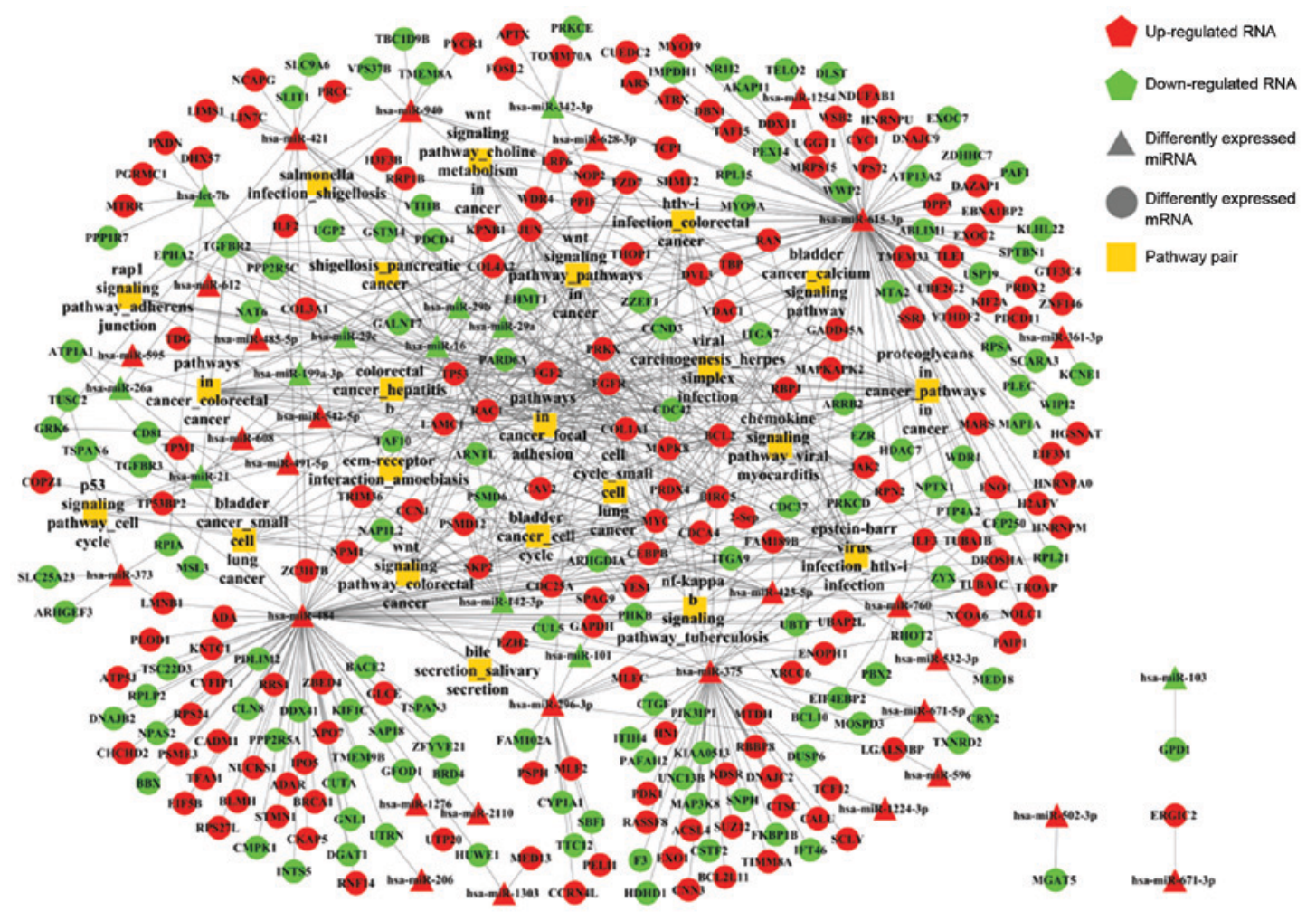

Figure 3. miRNA-mRNA-pathway pair complex network. miRNA, microRNA.

focal adhesion, proteoglycans in cancer and the Wnt signaling pathway.

Subsequently, 15 risk genes with the highest degrees were extracted in the present study and their topological properties are presented in Table III. Prediction accuracy of these 15 risk genes for NPC was measured by 10 -fold cross-validation, the AUC values for which are presented in Table IV. Among these risk genes, v-myc avian myelocytomatosis viral oncogene homolog (MYC), as a hub gene connecting with both miRNA and pathway pairs, serves the most important role in the miRNA-mRNA-pathway network (Fig. 4). 
Table IV. Prediction accuracy of 15 risk genes for NPC after 10-fold cross-validation.

\begin{tabular}{lcccc}
\hline Risk gene & AUC in GSE12452 & AUC in GSE34573 & AUC in GSE53819 & AUC in GSE13597 \\
\hline BCL2 & 0.829 & 1 & 0.5278 & 0.68 \\
BIRC5 & 0.8903 & 0.6 & 0.7901 & 0.9467 \\
CDC42 & 0.7 & 0.5778 & 0.7377 & 0.64 \\
EGFR & 0.6258 & 0.6 & 0.6975 & 0.6 \\
EZR & 0.9774 & 1 & 0.8179 & 0.7333 \\
FGF2 & 0.8548 & 0.7778 & 0.5617 & 0.8667 \\
JAK2 & 0.7806 & 0.9111 & 0.5895 & 0.68 \\
JUN & 0.6258 & 0.6444 & 0.8827 & 0.68 \\
MAPK8 & 0.8581 & 0.5333 & 0.8025 & 0.72 \\
MYC & 0.8645 & 0.9778 & 0.8302 & 0.8133 \\
PSMD12 & 0.8323 & 0.8 & 0.8981 & 0.76 \\
RAC1 & 0.6226 & 0.6 & 0.8272 & 0.92 \\
SKP2 & 0.9323 & 0.9111 & 0.7994 & 0.9733 \\
TBP & 0.6903 & 0.6 & 0.5123 & 0.6267 \\
TP53 & 0.7645 & 0.6914 & 0.8 \\
\hline
\end{tabular}

${ }^{a}$ Highest degree. The performance of the selected 15 risk genes for the NPC was evaluated using the 10-fold cross-validation through calculating the area under the receiver-operating characteristic curve. AUC, area under the curve; NPC, nasopharyngeal carcinoma.

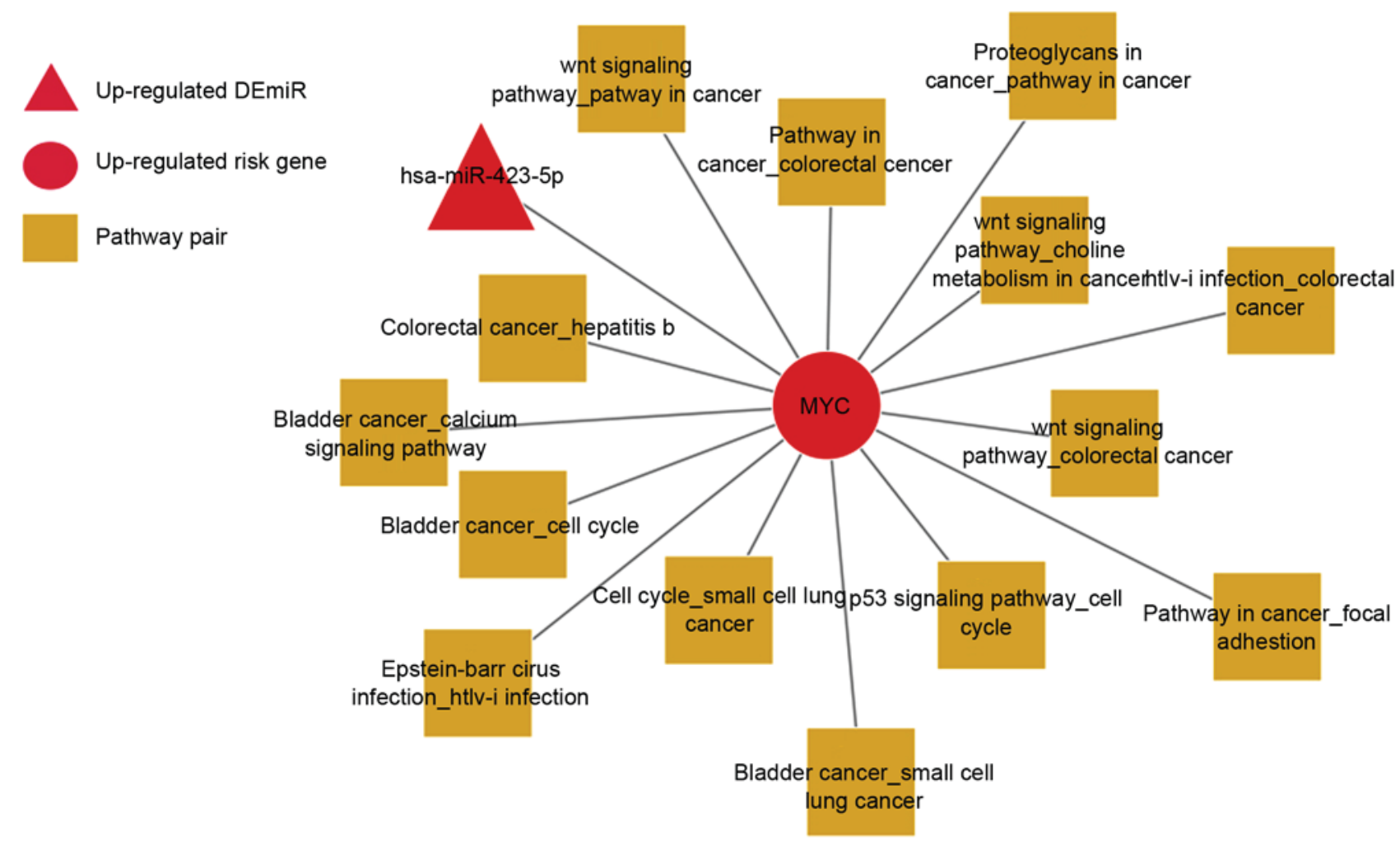

Figure 4. miRNA-mRNA-pathway pair network of MYC. Triangle indicates miRNA, circular indicates mRNA, yellow box indicates pathway pairs; red represents the up-regulated miRNAs, genes or pathway pairs. miRNA, microRNA; MYC, v-myc avian myelocytomatosis viral oncogene homolog.

Cross-validation of risk genes and its corresponding miRNA. MYC is differentially expressed in NPC. The AUC of MYC in GSE12452, GSE13597, GSE34573 and GSE53819 were $0.8645,0.8133,0.5333$ and 0.8302 , respectively. As presented in Fig. 4, MYC regulates 14 pathway pairs, including pathways in cancer_focal adhesion, proteoglycans in cancer_pathways in cancer, and Wnt signaling pathway_colorectal cancer. Additionally, focal adhesion has the highest degrees in the network, which is speculated to be associated with the development of NPC. 
In addition, MYC is regulated by hsa-miR-423-5p, which is differentially expressed in NPC. The AUC for hsa-miR-423-5p is 0.7798 .

\section{Discussion}

In order to improve understanding of the pathogenesis of NPC, the high-throughput sequencing datasets for NPC in EMBL-EBI were analyzed. A total of 1,540 DEGs and 218 DEmiRs were identified in the present study (699 upregulated genes and 841 downregulated genes; 192 upregulated miRNAs and 26 downregulated miRNAs). Genome-wide miRNA-, mRNA- and pathway-expression in the NPC was subsequently investigated, and the miRNA-mRNA-pathway network in NPC was constructed. Considering the topological properties of nodes in the network, the risk gene (MYC) with the highest degree was selected, which was indicated to serve an important role in NPC. Additionally, hsa-miR-423-5p, which is differentially expressed in NPC, was identified to regulate MYC.

In the present study, the focal adhesion pair pathway was significantly associated with NPC. As previously described, integrin cluster signaling is involved in cell migration and tumor invasion and focal adhesion kinase (FAK) is the key molecule in this signaling pathway (25). Suppressing the expression of FAK may retard cell invasion and redistribute the actin cytoskeleton to resemble a rounded dormant cell (26). Furthermore, re-expression of FAK may restore the motile phenotypes in FAK-negative cells (26). The focal adhesion pathway has previously been demonstrated to be involved in NPC carcinogenesis and progression (27,28). Additionally, Kassis et al (29) indicated that the focal adhesion pathway serves an important role in promoting EBV-associated invasiveness of NPC. EBV infection (a potential etiologic factor underlying NPC) is able to increase phosphorylation of FAK. The present study was consistent with previous discoveries and demonstrated the important role of focal adhesion in cancer, particularly in NPC.

Following extraction of information based on the topological properties from the miRNA-mRNA-pathway network, the risk gene MYC was observed to have the highest degree. It is well-accepted that activated signaling of MYC genes is a hallmark of several types of cancer, which also contributes to tumorigenesis by promoting cell growth, metastasis and angiogenesis (30). Additionally, MYC genes acting as transcription factors are able to modulate the transcription and expression of their target genes through various mechanisms $(31,32)$. MYC mutation or overexpression is associated with tumorigenesis $(33,34)$. In the present study, aberrantly-expressed MYC was identified with a prediction accuracy of 0.7568 . Previous studies have demonstrated that MYC genes are involved in the tumor progression and metastasis of NPC $(33,34)$, which was also indicated in the present study.

In addition, the MYC gene transcriptional network has been indicated to include miRNAs (35). Certain miRNAs, including those belonging to the oncogenic miR-17-92 cluster, have been demonstrated to be involved in MYC signaling (36-39). In the present study, a more general insight into the association between miRNAs and mRNAs within the MYC transcriptional network was provided. From the
miRNA-mRNA-pathways network, hsa-miR-423-5p, a novel biomarker targeting MYC, has been identified. Following 10-fold cross-validation, the prediction accuracy for classification effects of hsa-miR-423-5p was 0.7798. Studies have demonstrated that hsa-miR-423-5p is able to promote autophagy of tumor cells (40), which may also be considered a potential biomarker for cancer $(41,42)$. Based on the results of the present study, the targeting of MYC by hsa-miR-423-5p may serve an important role in the tumorigenesis, progression and metastasis of NPC, however, further experimental studies are required to identify their exact mechanisms in NPC.

In conclusion, MYC and hsa-miR-423-5p were identified to be potential miRNA and mRNA signatures of NPC, respectively. In addition, focal adhesion was indicated as one of the most important signaling pathways in the progression of NPC. The results provide molecular candidates for further studies into the diagnosis and treatment of NPC.

\section{References}

1. Lai SZ, Li WF, Chen L, Luo W, Chen YY, Liu LZ, Sun Y, Lin AH, Liu MZ and Ma J: How does intensity-modulated radiotherapy versus conventional two-dimensional radiotherapy influence the treatment results in nasopharyngeal carcinoma patients? Int J Radiat Oncol Biol Phys 80: 661-668, 2011.

2. Chou J, Lin YC, Kim J, You L, Xu Z, He B and Jablons DM: Nasopharyngeal carcinoma-review of the molecular mechanisms of tumorigenesis. Head Neck 30: 946-963, 2008.

3. He L and Hannon GJ: MicroRNAs: Small RNAs with a big role in gene regulation. Nat Rev Genet 5: 522-531, 2004.

4. Chen J, Zhang D, Zhang W, Tang Y, Yan W, Guo L and Shen B: Clear cell renal cell carcinoma associated microRNA expression signatures identified by an integrated bioinformatics analysis. J Transl Med 11: 169, 2013.

5. Lerebours F, Cizeron-Clairac G, Susini A, Vacher S, Mouret-Fourme E, Belichard C, Brain E, Alberini JL, Spyratos F, Lidereau $\mathrm{R}$ and Bieche I: miRNA expression profiling of inflammatory breast cancer identifies a 5-miRNA signature predictive of breast tumor aggressiveness. Int J Cancer 133: 1614-1623, 2013.

6. Pignot G, Cizeron-Clairac G, Vacher S, Susini A, Tozlu S, Vieillefond A, Zerbib M, Lidereau R, Debre B, Amsellem-Ouazana D and Bieche I: microRNA expression profile in a large series of bladder tumors: Identification of a 3-miRNA signature associated with aggressiveness of muscle-invasive bladder cancer. Int J Cancer 132: 2479-2491, 2013.

7. Xia H, Ng SS, Jiang S, Cheung WK, Sze J, Bian XW, Kung HF and Lin MC: miR-200a-mediated downregulation of ZEB2 and CTNNB1 differentially inhibits nasopharyngeal carcinoma cell growth, migration and invasion. Biochem Biophys Res Commun 391: 535-541, 2010.

8. Sengupta S, Den Boon JA, Chen IH, Newton MA, Dahl DB Chen M, Cheng YJ, Westra WH, Chen CJ, Hildesheim A, et al: Genome-wide expression profiling reveals EBV-associated inhibition of $\mathrm{MHC}$ class I expression in nasopharyngeal carcinoma. Cancer Res 66: 7999-8006, 2006.

9. Liu X, Lv XB, Wang XP, Sang Y, Xu S, Hu K, Wu M, Liang Y, Liu P, Tang J, et al: MiR-138 suppressed nasopharyngeal carcinoma growth and tumorigenesis by targeting the CCND1 oncogene. Cell Cycle 11: 2495-2506, 2012.

10. Zhang W, Zang J, Jing X, Sun Z, Yan W, Yang D, Shen B and Guo F: Identification of candidate miRNA biomarkers from miRNA regulatory network with application to prostate cancer. J Transl Med1 2: 66, 2014.

11. Yoon $\mathrm{S}$ and De Micheli G: Prediction of regulatory modules comprising microRNAs and target genes. Bioinformatics 21 (Suppl 2): ii93-ii100, 2005.

12. Bandyopadhyay S, Mitra R, Maulik U and Zhang MQ: Development of the human cancer microRNA network. Silence 1: 6,2010 .

13. Zhang W, Edwards A, Fan W, Flemington EK and Zhang K: miRNA-mRNA correlation-network modules in human prostate cancer and the differences between primary and metastatic tumor subtypes. PLoS One 7: e40130, 2012. 
14. Zhang S, Li Q, Liu J and Zhou XJ: A novel computational framework for simultaneous integration of multiple types of genomic data to identify microRNA-gene regulatory modules. Bioinformatics 27: i401-i409, 2011.

15. Kim SJ, Ha JW and Zhang BT: Constructing higher-order miRNA-mRNA interaction networks in prostate cancer via hypergraph-based learning. BMC Sys Biol 7: 47, 2013.

16. Ross DT, Scherf U, Eisen MB, Perou CM, Rees C, Spellman P, Iyer V, Jeffrey SS, Van de Rijn M, Waltham M, et al: Systematic variation in gene expression patterns in human cancer cell lines. Nat Genet 24: 227-235, 2000 .

17. Ihmels J, Friedlander G, Bergmann S, Sarig O, Ziv Y and Barkai N: Revealing modular organization in the yeast transcriptional network. Nat Genet 31: 370-377, 2002.

18. Segal E, Shapira M, Regev A, Pe'er D, Botstein D, Koller D and Friedman N: Module networks: Identifying regulatory modules and their condition-specific regulators from gene expression data. Nat Genet 34: 166-176, 2003.

19. Li J and Tseng GC: An adaptively weighted statistic for detecting differential gene expression when combining multiple transcriptomic studies. Annals Applied Statistics 5: 994-1019, 2011.

20. Ashburner M, Ball CA, Blake JA, Botstein D, Butler H, Cherry JM, Davis AP, Dolinski K, Dwight SS, Eppig JT, et al Gene ontology: Tool for the unification of biology. The gene ontology consortium. Nat Genet 25: 25-29, 2000.

21. Aoki-Kinoshita KF. and Kanehisa M: Gene annotation and pathway mapping in KEGG. In 'Comparative Genomics Volume 2' (Bergman, N.H., ed.), Humana Press, Methods Mol. Biol. 396, 71-92, 2007.

22. Huang DW, Sherman BT, Tan Q, Collins JR, Alvord WG, Roayaei J, Stephens R, Baseler MW, Lane HC and Lempicki RA: The DAVID Gene Functional Classification Tool: A novel biological module-centric algorithm to functionally analyze large gene lists. Genome Biol 8: R183, 2007.

23. Vigneron A, Gao LX, Golin MJ, Italiano GF and Li B: An algorithm for finding a k-median in a directed tree. Information Processing Lett 74: 81-88, 2000.

24. Shannon P, Markiel A, Ozier O, Baliga NS, Wang JT, Ramage D, Amin N, Schwikowski B and Ideker T: Cytoscape: A software environment for integrated models of biomolecular interaction networks. Genome Res 13: 2498-2504, 2003.

25. Parsons JT, Martin KH, Slack JK, Taylor JM and Weed SA: Focal adhesion kinase: A regulator of focal adhesion dynamics and cell movement. Oncogene 19: 5606-5613, 2000

26. Sieg DJ, Hauck CR and Schlaepfer DD: Required role of focal adhesion kinase (FAK) for integrin-stimulated cell migration J Cell Sci 112: 2677-2691, 1999.

27. Xu YF, Li YQ, Guo R, He QM, Ren XY, Tang XR, Jia WH, Kang TB, Zeng MS, Sun Y, et al: Identification of miR-143 as a tumour suppressor in nasopharyngeal carcinoma based on microRNA expression profiling. Int $\mathrm{J}$ Biochem Cell Biol 61 120-128, 2015.

28. Ou J, Pan F, Geng P, Wei X, Xie G, Deng J, Pang X and Liang H: Silencing fibronectin extra domain a enhances radiosensitivity in nasopharyngeal carcinomas involving an FAK/Akt/JNK Pathway. Int J Radiat Oncol Biol Phys 82: e685-e691, 2012.
29. Kassis J, Maeda A, Teramoto N, Takada K, Wu C, Klein G and Wells A: EBV-expressing AGS gastric carcinoma cell sublines present increased motility and invasiveness. Int J Cancer 99: 644-651, 2002

30. Adhikary S and Eilers M: Transcriptional regulation and transformation by Myc proteins. Nat Rev Mol Cell Biol 6: 635-645, 2005.

31. Nagesh N, Raju G, Srinivas R, Ramesh P, Reddy MD and Reddy ChR: A dihydroindolizino indole derivative selectively stabilizes G-quadruplex DNA and down-regulates c-MYC expression in human cancer cells. Biochim Biophys Acta 1850: 129-140, 2015.

32. Škunca Ž, Domimis M, Plninc-Peraica A and Jakšić B: Clinical features in DLBCL and translocation BCL2/c-MYC 'double hit' lymphoma. Acta Medica Croatica 68: 299-305, 2015 (In Croatian).

33. Yu X, Zhen Y, Yang H, Wang H, Zhou Y, Wang E, Marincola FM, Mai C, Chen Y, Wei H, et al: Loss of connective tissue growth factor as an unfavorable prognosis factor activates miR-18b by $\mathrm{PI} 3 \mathrm{~K} / \mathrm{AKT} / \mathrm{C}-\mathrm{Jun}$ and C-Myc and promotes cell growth in nasopharyngeal carcinoma. Cell Death Dis 4: e634, 2013.

34. Zhou W, Feng X, Ren C, Jiang X, Liu W, Huang W, Liu Z, Li Z, Zeng L, Wang L, et al: Over-expression of BCAT1, a c-Myc target gene, induces cell proliferation, migration and invasion in nasopharyngeal carcinoma. Mol Cancer 12: 53, 2013.

35. Bartel DP: MicroRNAs: Target recognition and regulatory functions. Cell 136: 215-233, 2009.

36. Chang TC, Yu D, Lee YS, Wentzel EA, Arking DE, West KM, Dang CV, Thomas-Tikhonenko A and Mendell JT: Widespread microRNA repression by Myc contributes to tumorigenesis. Nat Genet 40: 43-50, 2008.

37. Dews M, Homayouni A, Yu D, Murphy D, Sevignani C, Wentzel E, Furth EE, Lee WM, Enders GH, Mendell JT and Thomas-Tikhonenko A: Augmentation of tumor angiogenesis by a Myc-activated microRNA cluster. Nat Genet 38: 1060-1065, 2006.

38. Fontana L, Fiori ME, Albini S, Cifaldi L, Giovinazzi S, Forloni M, Boldrini R, Donfrancesco A, Federici V, Giacomini P, et al: Antagomir-17-5p abolishes the growth of therapy-resistant neuroblastoma through p21 and BIM. PLoS One 3: e2236, 2008.

39. O'Donnell KA, Wentzel EA, Zeller KI, Dang CV and Mendell JT: c-Myc-regulated microRNAs modulate E2F1 expression. Nature 435: 839-843, 2005.

40. Stiuso P, Potenza N, Lombardi A, Ferrandino I, Monaco A, Zappavigna S, Vanacore D, Mosca N, Castiello F, Porto S, et al: MicroRNA-423-5p promotes autophagy in cancer cells and is increased in serum from hepatocarcinoma patients treated with sorafenib. Mol Ther Nucleic Acids 4: e233, 2015.

41. McDermott AM, Miller N, Wall D, Martyn LM, Ball G, Sweeney KJ and Kerin MJ: Identification and validation of oncologic miRNA biomarkers for luminal a-like breast Cancer. PLoS One 9: e87032, 2014.

42. Hatse S, Brouwers B, Dalmasso B, Laenen A, Kenis C, SchöffskiP and Wildiers $\mathrm{H}$ : Circulating MicroRNAs as easy-to-measure aging biomarkers in older breast cancer patients: Correlation with chronological age but not with fitness/frailty status. PLoS One 9: e110644, 2014. 